Paola Letardi ${ }^{1}$, Monica Albini², ${ }^{2}$, Lidia mathys ${ }^{2,3}$, Mathilde Monachon ${ }^{3}$, Edith Joseph ${ }^{3,4}$

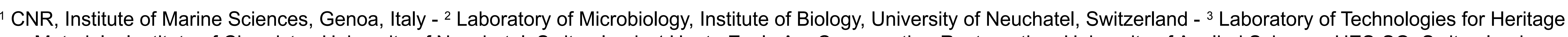
Materials, Institute of Chemistry, University of Neuchatel, Switzerland - 4 Haute Ecole Arc Conservation-Restauration, University of Applied Sciences HES-SO, Switzerland.

Protective treatments to be applied in the field of cultural heritage need to fulfil several constraints that have gradually been better outlined over the years. Along with the impact on aesthetic appearance and reversibility, it is nowadays well recognised that patinas and/or original historical coatings and finishings can play a relevant role in protective treatments' behaviour. Nonetheless, no standard characterisation method has been drafted to test new coatings and their application on real artefacts.

Electrochemical Impedance Spectroscopy (EIS) is a well established technique in the field of coating testing. In the latest decades the development of setups applicable on metal artworks has also opened the way to its use in the field of cultural heritage [1-4].

Contact Probe EIS measurements have been used all along the development of a bio-passivation method based on several years of research [5-8].
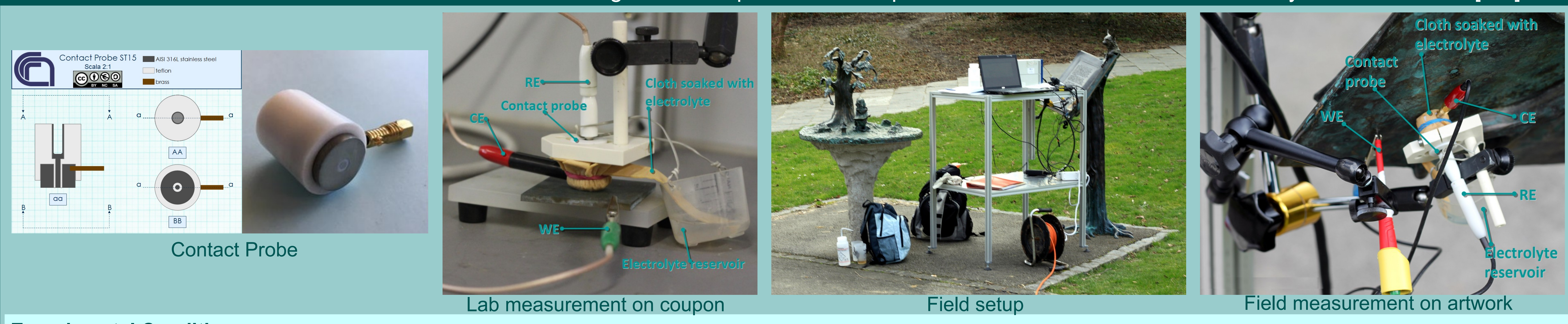

Experimental Conditions:

Gamry REF600 potentiostat; 30min stabilisation time; potentiostatic mode; $10 \mathrm{mV}$ at Eoc, range $100 \mathrm{KHz}-10 \mathrm{mHz} ; 10$ or 5 pts/decade.

\section{Model samples}

$\cdot 3$ bronze alloys

- 5 artificial patinas

$\cdot 3$ natural patinas (urban: Neuchatel; marine: Brest \& Genoa)

- 3 system tested (untreated T0, wax cosmoloid H80 TR, bio-based method T4)

$\cdot 12$ samples per set

Characterisation

Colorimetry

Fourier Transformed Infrared

Spectroscopy - FTIR

- Electrochemical Im

Spectroscopy - EIS

Energy Dispersive

SEM-EDS
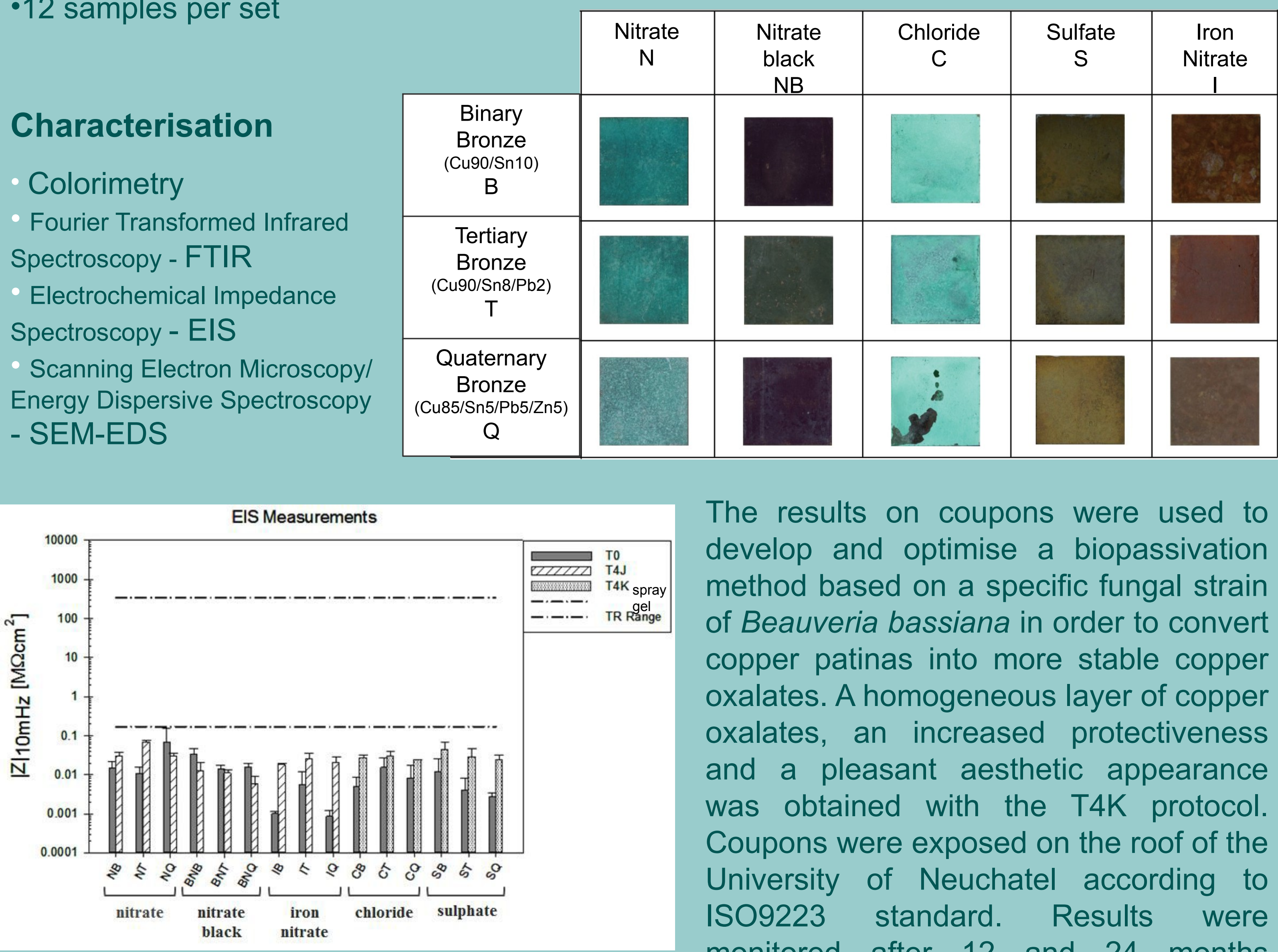

After ageing, $|Z|$ values of TR remained stable even if its thickness decreased. The $|Z|$ values from T4J and T4K remained mostly steady over time while the untreated samples showed an increase of the impedance values. This indicates a certain stability of biopassivated samples and instability of the artificial patinas that are still reaching a state of equilibrium with the exposure environment.

The results on coupons were used to evelop and optimise a biopassivation method based on a specific fungal strain of Beauveria bassiana in order to convert copper patinas into more stable copper oxalates. A homogeneous layer of copper oxalates, an increased protectiveness and a pleasant aesthetic appearance was obtained with the T4K protocol. Coupons were exposed on the roof of the University of Neuchatel according to ISO9223 standard. Results were monitored after 12 and 24 months

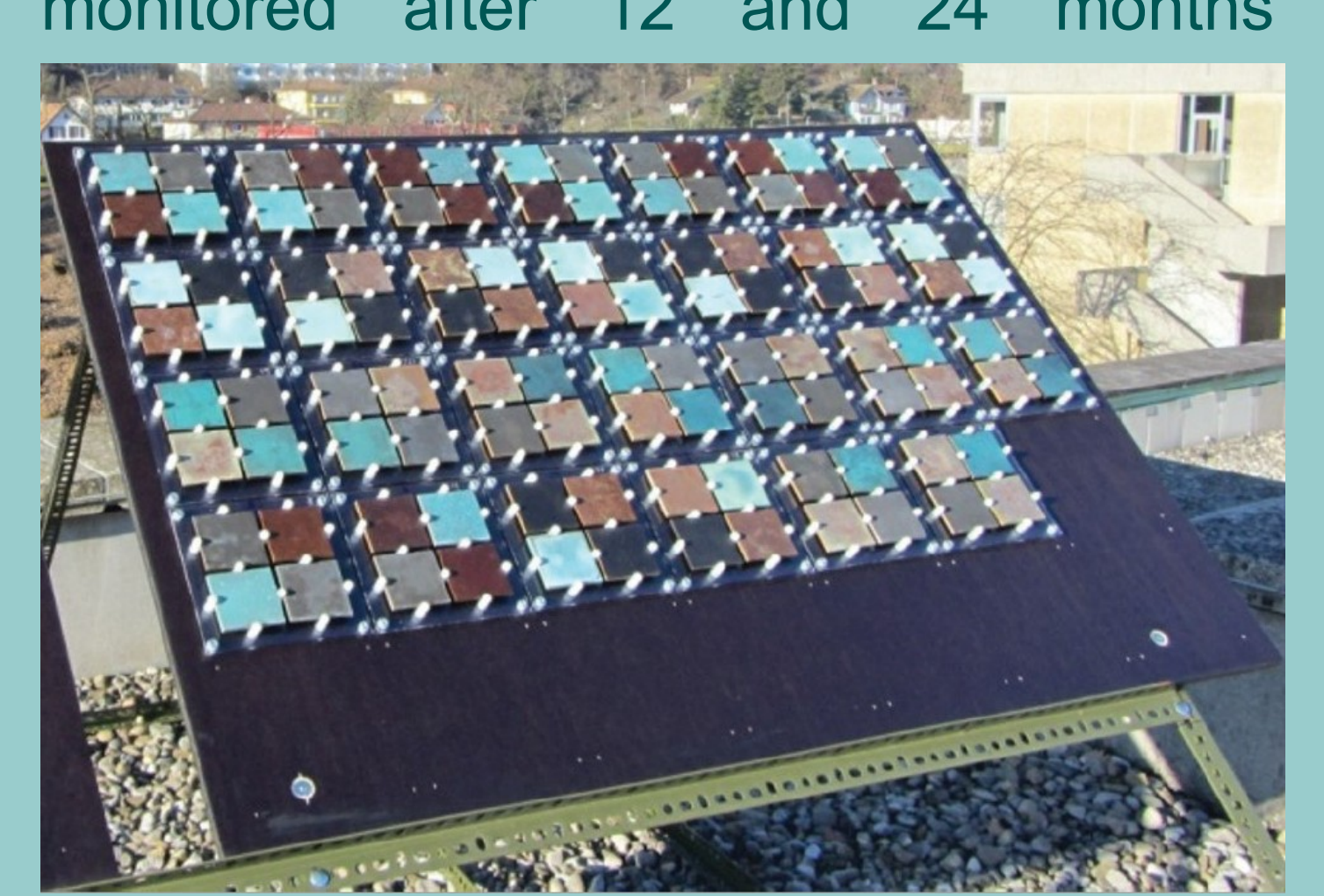

low conductivity electrolyte: mineral water $(300 \mu \mathrm{S} / \mathrm{cm})$ or artificial rain $10 \mathrm{X}(130 \mu \mathrm{S} / \mathrm{cm})$.

Pilot tests

In April 2014 a collaboration was initiated between the University of Neuchâtel and the Association "Legende d'Automne". The sculpture park "La Légende" is located in the heart of the Lausanne city measurements were performed after 24 months of exposure.
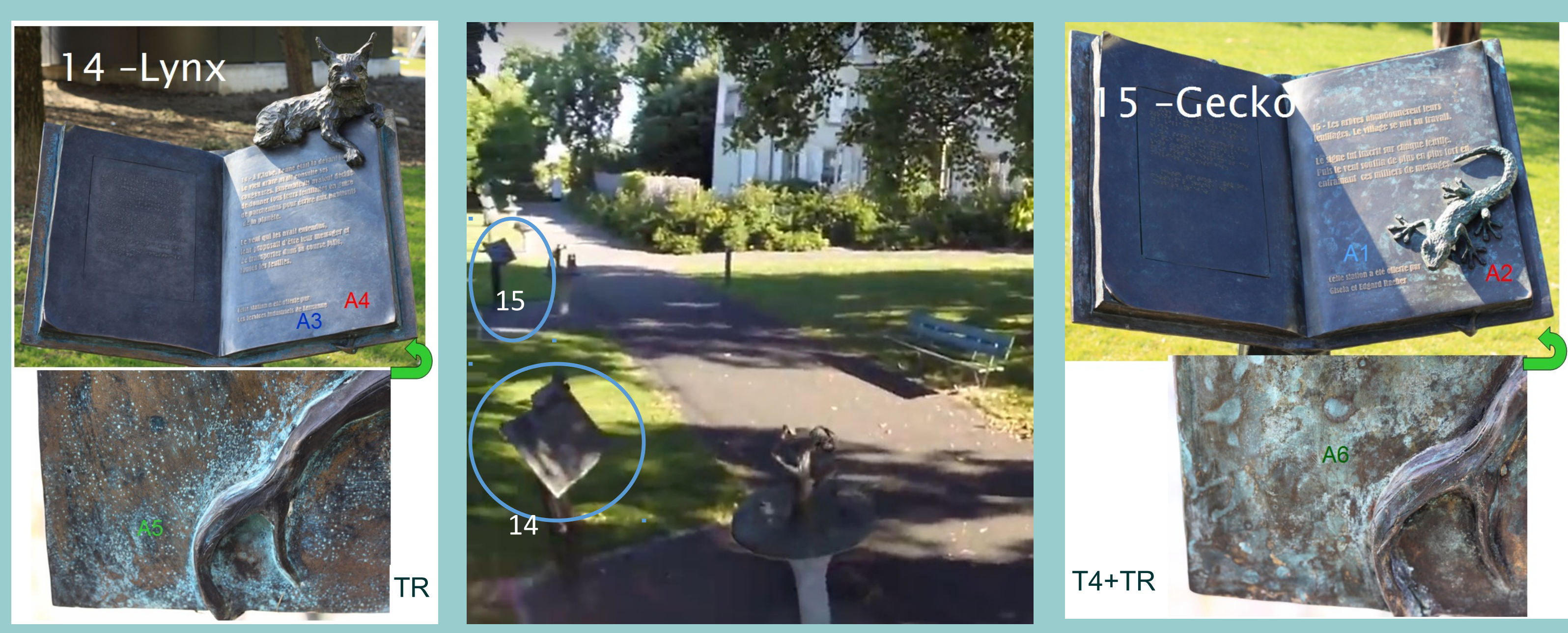

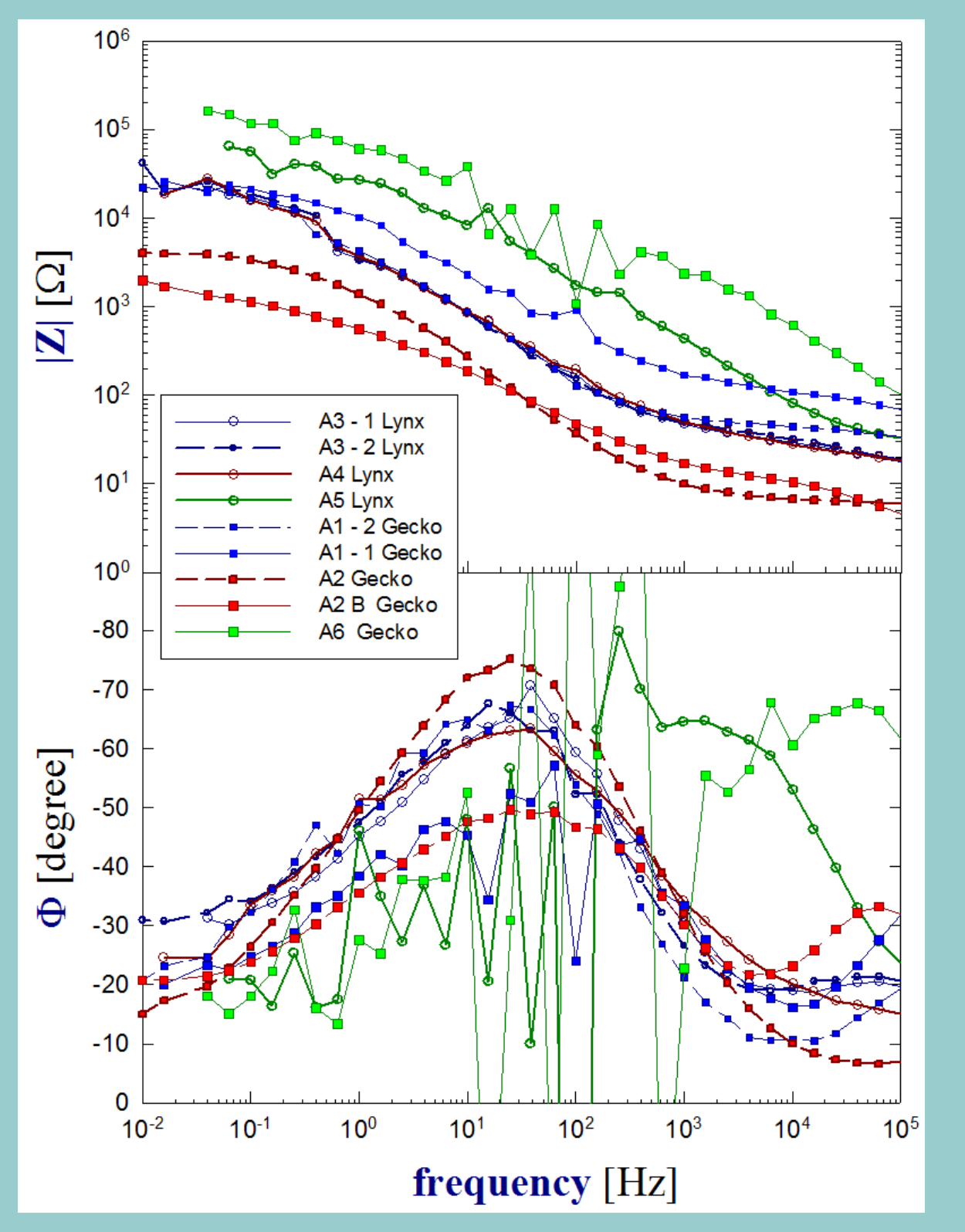

Similar EIS results are obtained on the front side (A1 A4) of the two measured lecterns. On the back side (A5-A6) both the high phase value at high frequency and the modulus values suggest a better behaviour of the T4+TR treated surface than the TR one. The colorimetric measurements highlighted a more homogenous surface appearance of the treated lecterns after 24 months for both treatments. However a difference was observed between the runoff (front side) and sheltered (back side) areas of the lecterns, with a ower colour variation for T4+TR.

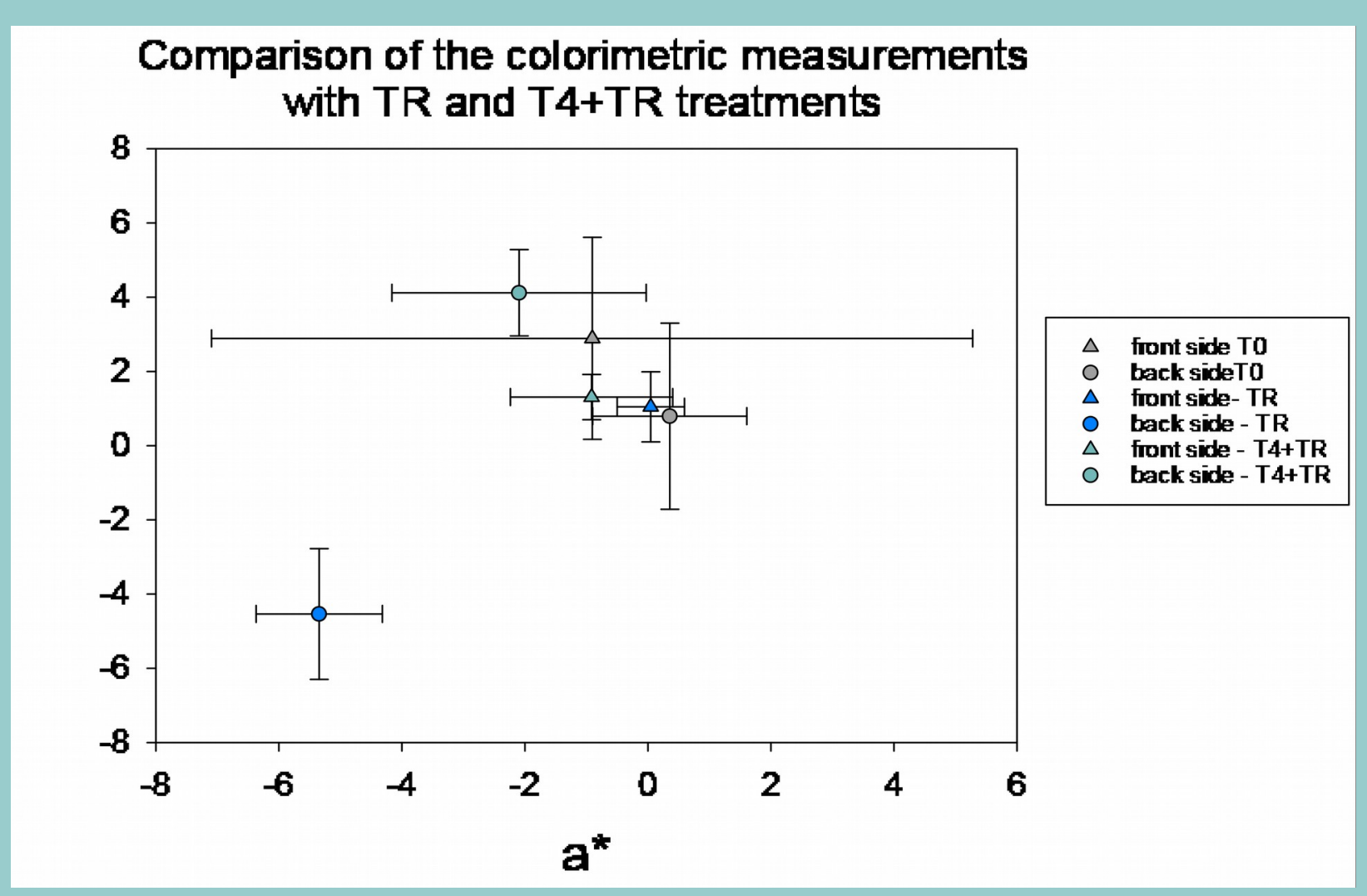

Bio-passivation was effective in the conversion of incohesive patinas into stable copper oxalates layers, avoiding the leach out of toxic products in the environment.

Contact Probe EIS measurements allowed to test and evaluate conservation treatments performance from coupon testing to real artefacts in their exposure environment.

Further improvements are in development to better exploit the valuable information in EIS spectra to support the decision making process of artwork preservation about best conservation treatments and monitoring. 